\title{
Complexity Results on Graphs with Few Cliques
}

\author{
Bill Rosgen ${ }^{1}$ and Lorna Stewart ${ }^{2}$ \\ ${ }^{1}$ Institute for Quantum Computing and School of Computer Science, University of Waterloo, Waterloo, Ontario, \\ Canada \\ ${ }^{2}$ Department of Computing Science, University of Alberta, Edmonton, Alberta, Canada
}

received Oct 21, 2005, revised June 25, 2007, accepted July 4, 2007.

\begin{abstract}
A graph class has few cliques if there is a polynomial bound on the number of maximal cliques contained in any member of the class. This restriction is equivalent to the requirement that any graph in the class has a polynomial sized intersection representation that satisfies the Helly property. On any such class of graphs some problems that are NP-complete on general graphs, such as the maximum clique problem and the maximum weighted clique problem, admit polynomial time algorithms. Other problems, such as the vertex clique cover and edge clique cover problems remain NP-complete on these classes. Several classes of graphs which have few cliques are discussed, and the complexity of some partitioning and covering problems are determined for the class of all graphs which have fewer cliques than a given polynomial bound.
\end{abstract}

Keywords: graphs, few cliques, Helly property, intersection representation, complexity

\section{Introduction}

All graphs we consider are finite, simple, and undirected, and for a graph $G=(V, E)$ we use $n=|V|$ and $m=|E|$ to refer to the number of vertices and edges of the graph. The distance between any two vertices $u$ and $v$ of a graph is the length of a shortest path between them, and is denoted $d(u, v)$. We set $d(u, v)=\infty$ if there is no path from $u$ to $v$. A clique in a graph is a subgraph in which every two vertices are adjacent. A clique is maximal if it is not contained in a larger clique. We use $K_{n}$ to denote the graph consisting of a single clique on $n$ vertices and $\mathcal{K}(G)$ to denote the set of all maximal cliques in $G$. For graphs $G_{1}$ and $G_{2}$ with disjoint vertex sets, we define the graph union of $G_{1}$ and $G_{2}$, denoted $G_{1} \cup G_{2}$, as the graph consisting of $G_{1}$ and $G_{2}$, without any connecting edges between them. For a graph $G$, and $t \in \mathbb{N}$ we define, for $t>1, t G=G \cup(t-1) G$, where $1 G=G$. For any positive integer $t$, we refer to the graph $\overline{t K_{2}}$ as $O_{t}$; these graphs were called octahedral graphs in [16].

Two sets are said to overlap if they intersect, but neither set is contained in the other. An intersection (respectively, overlap) representation of a graph is an assignment of sets to the vertices of the graph such that two vertices are adjacent if and only if the sets assigned to them intersect (respectively, overlap). The size of an intersection or overlap representation is simply the number of elements in the union of all sets in the collection. The size of a minimum intersection or overlap representation for a graph is known as the 
intersection number or overlap number of the graph, respectively. A family of sets has the Helly property if, for any subfamily that intersects pairwise, there is an element in common to all sets of the subfamily. We refer to an intersection or overlap representation that satisfies this property as a Helly intersection representation or a Helly overlap representation. The Helly intersection (respectively, overlap) number of a graph is the minimum size of a Helly intersection (respectively, overlap) representation.

For complete definitions of the graph classes mentioned here, see [3]. An exposition of the concept of NP-completeness, as well as some of the problems and hardness results used later, can be found in [7].

Moon and Moser, in their 1965 paper [15], show that $3^{\lceil n / 3\rceil}$ is the maximum number of maximal cliques in a graph on $n$ vertices, and observe that this bound is attained by the graph $\overline{(n / 3) K_{3}}$.

In this paper, we study classes of graphs that have a polynomial bound on the number of maximal cliques. A graph class is said to have few cliques or to be a few-cliques graph class if there is a polynomial $p(n)$ such that any $n$-vertex graph in the class has no more than $p(n)$ maximal cliques [16].

We focus on the complexities of problems on graph classes with few cliques. We are motivated in part by a connection between few-cliques graph classes and intersection and overlap graphs. It has long been known that chordal graphs [11], Helly circular-arc (respectively, circle) graphs [10, 5], and boxicity 2 graphs (see [18]) have Helly intersection representations of size polynomial in the number of vertices in the graph. Given a Helly intersection representation of polynomial size for a class of graphs, the maximum weight clique problem can be solved in polynomial time for the intersection class [18] as well as for the corresponding overlap graph class [4]. Thus since, as noted in [18] and discussed in more detail in Section 2 a polynomial bound on the Helly intersection number turns out to be equivalent to a polynomial bound on the number of maximal cliques in a graph class, the few-cliques classes are interesting within the context of intersection and overlap graphs. An additional motivation for our work is found in the study of relationships among graph parameters and algorithms, where it is interesting to ask which problems become tractable as a result of bounding a particular parameter and which problems remain as computationally difficult as for graphs in general.

\section{Graphs with Few Cliques}

By the definition, each of the following graph classes has few cliques: any finite graph class, complete graphs, and triangle-free graphs. In addition, since chordal graphs have at most $n$ maximal cliques [6], graphs of boxicity $k$ have at most $(2 n)^{k}$ maximal cliques [18], planar graphs have at most $7 n / 3-6$ maximal cliques [16], and $K_{k}$-free graphs have at $\operatorname{most} \max \left\{n, n \Delta^{k-2} / 2^{k-2}\right\}$ maximal cliques, where $\Delta$ is the maximum degree of any vertex in the graph [16], all of these graph classes have few cliques.

Balas and $\mathrm{Yu}$ [1] prove the following bound on the number of maximal cliques a graph can have, in terms of the largest induced subgraph of the form $O_{t}$.

Theorem 1 (Balas and Yu [1]) For any connected graph $G=(V, E)$, where $\delta$ is the number of pairs $(u, v) \in V \times V$ with $d(u, v)=2$, and $t$ is the largest integer such that $G$ contains an induced subgraph isomorphic to $O_{t}$, the number of cliques in the graph is bounded by

$$
2^{t} \leq|\mathcal{K}(G)| \leq \delta^{t}+1 .
$$

Note that if $t$ is bounded by some constant for a class of graphs, this theorem implies a polynomial bound on the number of maximal cliques in graphs in the class.

Another partial characterization of the graph classes with few cliques is shown by Prisner in [16], where he considers classes of graphs having $O_{t}$ as a forbidden induced subgraph, and derives upper bounds on 
the number of cliques in such graphs. A corollary to these bounds characterizes the hereditary classes of graphs which have few cliques.

Theorem 2 (Prisner [16]) A hereditary class of graphs $\mathcal{C}$ has few cliques if and only if for some constant $t, O_{t} \notin \mathcal{C}$.

A further characterization of graph classes with few cliques relies on the following notions related to hypergraphs, from [2] and [3]. A hypergraph $H=(V, \mathcal{E})$ is a set of vertices $V$ and hyperedges (or edges) $\mathcal{E}$, where each element of $\mathcal{E}$ is a nonempty subset of $V$. The dual of a hypergraph $H=(V, \mathcal{E})$ is hypergraph $H^{*}=\left(\mathcal{E},\left\{E_{v}: v \in V\right\}\right)$, where $E_{v}$ is the set of hyperedges in $\mathcal{E}$ that contain the vertex $v$. The 2-section, $(H)_{2}$, of a hypergraph $H=(V, \mathcal{E})$ is the graph $G=(V, E)$, where $(u, v) \in E$ if and only if there is an edge $e \in \mathcal{E}$ such that $u, v \in e$. We say that a hypergraph $H=(V, \mathcal{E})$ is Helly when the edge set $\mathcal{E}$ satisfies the Helly property. A hypergraph $H$ is conformal if each clique of $(H)_{2}$ is contained in an edge of $H$. Note that the 2-section of the dual of a hypergraph $H$ is the intersection graph of the hyperedges of $H$. Now, the following theorem can be found in [2], where it is attributed to Gilmore.

Theorem 3 (see Berge [2, page 396]) A hypergraph is Helly if and only if its dual is conformal.

Let $G=(V, E)$ be a graph and, for each vertex $v \in V$, let $S_{v}$ be the set of all maximal cliques of $G$ that contain $v .\left\{S_{v}: v \in V\right\}$ is an intersection representation for $G$ since vertices are adjacent if and only if they appear together in some maximal clique; it satisfies the Helly property since pairwise intersecting sets represent a clique and all vertices of any clique are contained in some maximal clique. Since the size of the representation is $|\mathcal{K}(G)|$, we have that the Helly intersection number of $G$ is at most $|\mathcal{K}(G)|$.

Furthermore, for an arbitrary Helly intersection representation of graph $G=(V, E)$, consider the hypergraph $H=\left(X,\left\{S_{v}: v \in V\right\}\right)$ where, for each $v \in V, S_{v}$ is the set representing $v$, and $X=$ $\cup_{v \in V} S_{v}$. By Theorem 3 , each maximal clique of $G=\left(H^{*}\right)_{2}$ is contained in an edge of $H^{*}$ and, combined with the fact that each edge of $H^{*}$ corresponds to a clique in $G$, this implies that $H^{*}$ has a unique edge for each maximal clique of $G$. Therefore, $|\mathcal{K}(G)|$ is less than or equal to the Helly intersection number of $G$.

Thus the next result, which has previously been observed, for example in [18], is an immediate corollary of Theorem 3

Corollary 4 For any graph $G$, the number of maximal cliques in $G$ is equal to the Helly intersection number of $G$.

\section{Complexity Observations}

The problem of recognizing a few-cliques graph class is, given some polynomial bound $p(n)$, does the input graph have no more than $p(n)$ maximal cliques? This problem is solvable in polynomial time by using an algorithm that enumerates all maximal cliques in a graph, such as one of the algorithms of [19] or [12]. Both of these algorithms have what is referred to in [12] as the polynomial delay property, that is, the length of time before the first clique is generated and the length of time between the algorithm generating any two successive cliques are each bounded by a polynomial in the size of the graph. Thus, we run the algorithm to enumerate the cliques, and within a polynomial amount of time the algorithm either generates too many cliques, whence we stop the computation and reject, or enumerates all of the cliques in the input graph, at which time the algorithm accepts. Thus, given a graph $G$ and a polynomial $p(n)$, the questions: does $G$ have no more than $p(n)$ maximal cliques? and does $G$ have Helly intersection number 
at most $p(n)$ ? can be answered in polynomial time. Furthermore, for yes instances of the problems, all maximal cliques can be enumerated, as described above, and a minimum Helly intersection representation can be constructed in polynomial time. As discussed in Section 2 a minimum Helly representation can be produced by generating all maximal cliques and then identifying the set of cliques that each vertex is contained in.

Note that polynomial time algorithms for graphs with few cliques can be made robust (that is, when given input not in the class of graphs the algorithm is designed for, the algorithm either rejects or continues computing the correct output [18]) by generating at most $p(n)+1$ cliques and indicating that the input was not in the class of graphs if more than $p(n)$ cliques are found.

In addition, the maximum clique and weighted maximum clique problems are trivially polynomially solvable for few-cliques graph classes. We first apply the algorithm in [19] to find all maximal cliques in time $O(n m p(n))$. Then, to solve the maximum clique problem we iterate over the list of all maximal cliques, selecting the largest one. A similar algorithm solves the vertex-weighted maximum clique problem, since a maximum weighted clique is some maximal clique with the negative weight vertices removed.

Our NP-completeness results are provided for the class of all graphs containing not more than $p(n)$ maximal cliques, for a given polynomial $p(n)$. Since the graph classes we have observed to have few cliques are contained in the class of graphs with not more than $p(n)$ cliques, for some $p(n)$, NP-completeness results for these classes directly imply the NP-completeness of the same problem on the class of graphs with not more than $p(n)$ cliques.

Spinrad [18] observed that boxicity-2 graphs have no more than $4 n^{2}$ cliques and, therefore, since Colourability, IndePendent Set, and Vertex Colour are NP-complete [14, 17] for boxicity-2 graphs, they are NP-complete for those graphs with no more than $4 n^{2}$ maximal cliques.

The NP-completeness of the Hamiltonian cycle and Hamiltonian path problems on planar graphs is shown in [9] and the dominating set problem on planar graphs is shown NP-complete in [7]. Thus, by the bound in [16] which limits the number of maximal cliques in a planar graph to $7 n / 3-6$ we know that these problems are NP-complete for the class of graphs with no more than $7 n / 3-6$ maximal cliques.

\section{Covering and Partitioning Problems}

In this section we consider the complexity of covering and partitioning problems under the restriction that the input graph has few cliques. Our results reveal that the hardness of many of these problems lies not only in choosing the cliques in a cover from an exponential collection, but in choosing those cliques that lead to a minimum cover.

In Section 3 , we saw that having a polynomial bound on the number of maximal cliques does not help to solve the problem of finding the minimum colouring of a graph; in this section, we consider the problem of colouring a graph with no more than some constant number of colours, $k$, where $k \geq 3$.

Problem. $k$-Colourability

Instance: A graph, $G=(V, E)$.

Question: Can the vertices of $G$ be coloured with $k$ or fewer colours such that adjacent vertices are assigned distinct colours? 
Theorem $5 k$-Colourability, for $k \geq 3$, is NP-complete on the class of graphs with no more than $7 n / 3-6$ maximal cliques.

Proof: 3-Colourability is known to be NP-complete on planar graphs [8], and thus, by the bound given in [16], the problem is also NP-complete for the class of graphs with no more than $7 n / 3-6$ maximal cliques. However, this result does not extend, on planar graphs, to values of $k$ larger than 3 , since it is known that any planar graph can be coloured using only four colours.

We use a simple reduction from the $k$-colouring problem to the $k+1$-colouring problem to form an inductive proof for graphs with no more than $7 n / 3-6$ maximal cliques. We take a graph $G=(V, E)$, an instance of the $k$-colourability problem, and create a graph $G^{\prime}$ by adding a single universal vertex. Note that $|\mathcal{K}(G)|=\left|\mathcal{K}\left(G^{\prime}\right)\right|$ and that $G$ is $k$-colourable if and only if $G^{\prime}$ is $k+1$-colourable, since a $k$-colouring for $G$ together with a new colour for the universal vertex is a $k+1$-colouring for $G^{\prime}$ and vice versa. Therefore, by induction on $k$, for $k \geq 3$, this demonstrates the NP-completeness of the $k$-colourability problem on the class of graphs with not more than $7 n / 3-6$ maximal cliques, for any constant $k \geq 3$.

We now examine the problem of partitioning the vertices of a graph into disjoint cliques of size $k$, for some constant $k$.

Problem. $k$-CliQue PARTITION

Instance: A graph, $G=(V, E)$.

Question: Can the vertices of the graph $G$ can be partitioned into cliques of size $k$ ?

This problem can be shown to be NP-complete, for any $k \geq 3$, by a reduction similar to the one used in the proof of Theorem 5. The case $k=3$ is done by Garey and Johnson in [7], where the exact cover by 3-sets problem is used to show the NP-completeness of 3-CLIQUE PARTITION (which they call the triangle partition problem), but most importantly for our purposes, the reduction they use produces a $K_{4}$ free graph. Thus, this result can be immediately extended to 3-CLIQUE PARTITION on graphs with no more than $p(n)$ cliques, for some cubic polynomial $p(n)$, since there are no more than $n^{3}$ cliques in a $K_{4}$ free graph.

Theorem $6 k$-Clique PARTITION, for $k \geq 3$, is NP-complete for the class of graphs with no more than $n^{k}$ maximal cliques.

Proof: We apply a simple transformation to reach from the case $k$ to the case $k+1$. For a graph $G=$ $(V, E)$ on $n$ vertices that is an instance of the $k$-clique partition problem, we construct the graph $G^{\prime}=$ $\left(V^{\prime}, E^{\prime}\right)$, where we obtain $G^{\prime}$ from $G$ by adding $n / k$ vertices, each connected to every vertex of $G$.

Notice that a graph cannot be partitioned into disjoint $k$-cliques unless $k$ divides $|V|=n$, and so, if this is not the case, we can simply output a trivial no instance, such as the one vertex graph, which cannot be partitioned into $k$-cliques for any $k>1$. If on the other hand, $k$ divides $n$, we construct $V^{\prime}$ and $E^{\prime}$ as

$$
\begin{aligned}
& V^{\prime}=V \cup\left\{w_{i}: 1 \leq i \leq n / k\right\} \\
& E^{\prime}=E \cup\left\{\left(w_{i}, v\right): v \in V, 1 \leq i \leq n / k\right\} .
\end{aligned}
$$

This can clearly be done in polynomial time. Notice first that, as long as we do not output the trivial no instance, we know that $k+1$ divides $\left|V^{\prime}\right|=n+n / k$. 
We prove that the reduction is correct by induction on $k$, the base case, for $k=3$ is the triangle partition problem [7]. For $k \geq 3$, we build a graph $G^{\prime}$ using the transformation given above. If we take a $k$-clique partition for $G$, we can extend it to a $k+1$ clique partition for $G^{\prime}$, as we must use $n / k$ cliques of size $k$ to partition $G$, so we can take one of the new vertices into each $k$ clique to form a $k+1$ clique. In the other direction, any $k+1$ partition of $G^{\prime}$ must use one of the new vertices in each clique, as no two of the new vertices are adjacent. Since there are exactly $n / k$ new vertices, and there are $(n+n / k) /(k+1)=n / k$ cliques in any $k+1$ clique partition of $G^{\prime}$, so we will use all of the newly added vertices. Thus, we can simply remove the new vertices from the clique partition to obtain a $k$-clique partition of $G$. Thus, by induction on $k$, this reduction reduces the 3-clique partition problem to the $k$-clique partition problem for all $k \geq 3$. Notice also that at each step of the induction we increase the number of maximal cliques by a factor of $n / k$. Hence, the number of cliques in the final graph is bounded by $n^{k-3} p(n)$, if $p(n)$ is a bound on the number of cliques in the instance of the 3-clique partition problem, and since one possible choice for $p(n)$ is $n^{3}$, as the initial instance of the triangle partition problem was $K_{4}$ free, the constructed instance of the $k$-clique partition problem has no more than $n^{k}$ cliques, which is polynomial in $n$, for fixed $k$. Thus we have shown the NP-completeness of the $k$-clique partition problem on the graphs with not more than $n^{k}$ maximal cliques.

We now consider the vertex clique partition problem.

Problem. Vertex Clique Partition

Instance: A graph, $G=(V, E)$, and a natural number $k$.

Question: Can the vertices of graph $G$ can be partitioned into $k$ or fewer cliques?

Note that the vertex clique partition and vertex clique cover problems are equivalent, since any subgraph of a clique is again a clique.

In light of the preceding results, we show that vertex clique partition problem is NP-complete by reducing an $n$-vertex $K_{4}$ free instance $G$ of 3-CLIQUe PARTITION to the instance $(G,\lfloor n / 3\rfloor)$ of VerTeX Clique Partition. $G$ can be partitioned into at most $\lfloor n / 3\rfloor$ cliques if and only if it can be partitioned into triangles, since the graph is $K_{4}$ free. A potential complication arises on an instance where 3 does not divide $n$, but such an instance cannot be partitioned into triangles, so the reduction can output an arbitrary no instance of the clique partition problem. We conclude that VerTex CLIQUe PARTITION for the class of graphs with no more than $n^{3}$ cliques is NP-complete.

In addition to the NP-completeness of Vertex Clique Cover, we also have the NP-completeness of EDGE CLIQUE COVER for graphs with a polynomially bounded number of maximal cliques. This result comes directly from a result in [13], where the vertex clique cover problem is reduced to the edge clique cover problem by taking an instance $G$ of VERTEX CLIQUe COVER, and adding $m+1$ vertices to the graph that are adjacent to all the vertices of $G$, where $m$ is the number of edges in $G$. If $G$ has no more than $p(n)$ maximal cliques, then the resulting instance of the edge clique cover problem has no more than $(m+1) p(n)$ maximal cliques. Thus, using this reduction, we have the NP-completeness of the edge clique cover problem, that is, the intersection number problem, for graphs with no more than $(m+1) n^{3}$ maximal cliques.

Finally, we encounter a problem that is solvable in polynomial time on graphs with few cliques. This problem is simply the $k$-colourability problem in the complement graph. 
Problem. Vertex Clique $k$-Partition

Instance: A graph, $G=(V, E)$.

Question: Can the vertices of graph $G$ can be partitioned into $k$ or fewer cliques?

Related to this problem are the problems of covering the vertices and edges of a graph with at most $k$ cliques. These problems can once again be obtained from the previous problem by allowing the vertices of the graph to be in multiple cliques in the cover. The essential idea behind a polynomial time algorithm for these problems on a graph $G$ with no more than $p(n)$ maximal cliques is that if we can cover a graph with cliques, we can also cover the graph with the same number of maximal cliques, by simply enlarging each of the cliques to some maximal clique. We can thus consider only collections of $k$ maximal cliques.

For graph $G=(V, E)$, we first compute $\mathcal{K}(G)=\left\{C_{1}, C_{2}, \ldots, C_{r}\right\}$ using the algorithm in [19], which runs in $O(n m r)$ time. Then we consider each of the $\left(\begin{array}{l}r \\ k\end{array}\right)$ sets of $k$ maximal cliques of $G$. If any of these forms a clique cover, we output yes. If none of these are clique covers of $G$, we output no. Checking if each set is a clique cover can be performed in $O\left(n^{2}\right)$ time, for a total runtime of $O\left(n^{2}\left(\begin{array}{l}r \\ k\end{array}\right)\right)=O\left(n^{2} p(n)^{k}\right)$ where $p(n)$ is a polynomial bound on the number of cliques, and so we have a polynomial time algorithm for the clique $k$-cover problem for graphs with a polynomially bounded number of maximal cliques.

Once again, by the equivalence of Vertex Clique Cover and Vertex Clique Partition, this algorithm solves the $k$-partitioning problem as well, and since we can form a minimum edge $k$-clique cover from maximal cliques by using the same approach, we have a polynomial time algorithm, for any class of graphs with few cliques, for the edge $k$-clique cover problem. This problem is exactly the problem of determining for a graph $G$ whether there is an intersection representation for $G$ of size at most $k$, for constant $k$ [13].

\section{Conclusion}

We have seen that the graphs with few cliques are exactly those graphs with polynomially bounded Helly intersection number.

We have also examined several well-known NP-complete graph problems, and shown that the majority of them remain hard when restricted to the graphs with few cliques.

Table 1 lists the complexity results on the problems considered here, for graphs with few cliques. It is interesting to note that the NP-completeness of edge clique cover implies that it remains hard to find a minimum intersection representation even when given a minimum Helly intersection representation.

The problems that admit efficient algorithms on these restricted classes appear to be only those problems where the restriction allows a brute force algorithm to run efficiently. Whether there is a problem that allows this restriction to be used in the construction of a nontrivial efficient algorithm is unknown.

\section{Acknowledgements}

This research was partially supported by NSERC and iCORE. 


\begin{tabular}{|c|c|c|}
\hline Problem & Complexity & Bound \\
\hline CLIQUE & $O(n m p(n))$ & \\
\hline COLOURABILITY & NP-complete & $4 n^{2}$ \\
\hline Dominating SET & NP-complete & $7 n / 3-6$ \\
\hline EDGE Clique COVER & NP-complete & $(m+1) n^{3}$ \\
\hline EdGe Clique $k$-COVER & $O\left(n^{2} p(n)^{k}\right)$ & \\
\hline HAMILTONIAN CYCLE & NP-complete & $7 n / 3-6$ \\
\hline HAMILTONIAN PATH & NP-complete & $7 n / 3-6$ \\
\hline INDEPENDENT SET & NP-complete & $4 n^{2}$ \\
\hline$k$-Clique PARTITION & NP-complete & $n^{k}$ \\
\hline$k$-COLOURABILITY & NP-complete & $7 n / 3-6$ \\
\hline Vertex Clique Cover & NP-complete & $n^{3}$ \\
\hline VERTEX Clique $k$-COVER & $O\left(n^{2} p(n)^{k}\right)$ & \\
\hline VERTEX CLIQUe $k$-PARTITION & $O\left(n^{2} p(n)^{k}\right)$ & \\
\hline VERTEX Clique PARTITION & NP-complete & $n^{3}$ \\
\hline VERTEX COVER & NP-complete & $4 n^{2}$ \\
\hline Weighted Clique & $O(n m p(n))$ & \\
\hline
\end{tabular}

Tab. 1: Complexity results on graphs with $n$ vertices, $m$ edges, and no more than $p(n)$ maximal cliques. The NP-completeness results hold for the class of graphs that do not have more maximal cliques than the given bound.

\section{References}

[1] E. Balas and C. S. Yu. On graphs with polynomially solvable maximum-weight clique problem. Networks, 19(2):247-253, 1989.

[2] C. Berge. Graphs and Hypergraphs. Number 6 in North-Holland Mathematical Library. NorthHolland, 1973.

[3] A. Brandstädt, V. B. Le, and J. P. Spinrad. Graph Classes: A Survey. SIAM Monographs on Discrete Mathematics and Applications. Society for Industrial and Applied Mathematics, 1999.

[4] E. Čenek and L. Stewart. Maximum independent set and maximum clique algorithms for overlap graphs. Discrete Applied Mathematics, 131(1):77-91, 2003.

[5] G. Durán. Some new results on circle graphs. Matemática Contemporânea, 25:91-106, 2003.

[6] D. R. Fulkerson and O. A. Gross. Incidence matrices and interval graphs. Pacific Journal of Mathematics, 15(3):835-855, 1965.

[7] M. Garey and D. Johnson. Computers and Intractability: A Guide to the Theory of NPCompleteness. W. H. Freeman and Company, 1979.

[8] M. R. Garey, D. S. Johnson, and L. Stockmeyer. Some simplified NP-complete problems. In Proceedings of the 6thAnnual ACM Symposium on Theory of Computing, pages 47-63, 1974. 
[9] M. R. Garey, D. S. Johnson, and R. E. Tarjan. The planar Hamiltonian circuit problem is NPcomplete. SIAM Journal on Computing, 5(4):704-714, 1976.

[10] F. Gavril. Algorithms on circular-arc graphs. Networks, 4:357-369, 1974.

[11] F. Gavril. The intersection graphs of subtrees in trees are exactly the chordal graphs. Journal of Combinatorial Theory, Series B, 16:47-56, 1974.

[12] D. S. Johnson, M. Yannakakis, and C. H. Papadimitriou. On generating all maximal independent sets. Information Processing Letters, 27(3):119-123, 1988.

[13] L. T. Kou, L. J. Stockmeyer, and C. K. Wong. Covering edges by cliques with regard to keyword conflicts and intersection graphs. Communications of the Association for Computing Machinery, 21(2):135-139, 1978.

[14] D. T. Lee and J. Y.-T. Leung. On the 2-dimensional channel assignment problem. IEEE Transactions on Computers, 33(1):2-6, 1984.

[15] J. W. Moon and L. Moser. On cliques in graphs. Israel Journal of Mathematics, 3:23-28, 1965.

[16] E. Prisner. Graphs with few cliques. In Proceedings of the 7th Quadrennial International Conference on the Theory and Applications of Graphs, 1992, Graph Theory, Combinatorics, and Applications, pages 945-956, 1995.

[17] C. S. Rim and K. Nakajima. On rectangle intersection and overlap graphs. IEEE Transactions on Circuits and Systems I: Fundamental Theory and Applications, 42(9):549-553, 1995.

[18] J. P. Spinrad. Efficient Graph Representations. Number 19 in Fields Institute Monographs. American Mathematical Society, 2003.

[19] S. Tsukiyama, M. Ide, H. Arioyoshi, and I. Shirakawa. A new algorithm for generating all the maximal independent sets. SIAM Journal on Computing, 6(3):505-517, 1977. 
